\title{
A retrospective study of the prognostic value of MRI-derived residual tumors at the end of intensity-modulated radiotherapy in 358 patients with locally-advanced nasopharyngeal carcinoma
}

\author{
Yuxiang He, Qin Zhou, Lin Shen, Yajie Zhao, Mingjun Lei, Rui Wei, Liangfang Shen ${ }^{*}$ and Shousong Cao
}

\begin{abstract}
Objective: To retrospectively analyze the prognostic value of magnetic resonance imaging (MRI)-derived residual tumors after intensity-modulated radiation therapy (IMRT) in the patients with locally-advanced nasopharyngeal carcinoma.

Methods: A total of 358 patients with locally-advanced nasopharyngeal carcinoma who received IMRT were classified as having residual tumors or no residual tumor based on MRI at the end of radiotherapy. The $x^{2}$ test, log-rank test, Cox proportional hazards regression model and Kaplan-Meir survival curves were used to investigate the relationship of clinicopathological features and residual tumors and to assess the prognostic value of residual tumors.

Results: The 3-year overall survival (OS) rate was $73 \%$ in the residual tumor group and $90 \%$ in the no residual tumor group (HR 2.15, 95\% Cl 1.21-3.82 "P=0.007); 3-year local relapse-free survival (LRFS) was $89 \%$ in the residual tumor group and $97 \%$ in the no residual tumor group (HR 4.46, 95\% Cl 1.61-12.38, $P=0.002$ ); 3-year disease free survival (DFS) was $67 \%$ in the residual tumor group and $82 \%$ in the no residual tumor group (HR 2.21,95\% Cl 1.40-3.48, $P=0.001$ ). A high prescribed radiation dose (>73.92 Gy) did not increase the percentage volume of the GTVnx receiving $95 \%$ of the prescribed dose (GTVnx V95\%) or improve any survival outcome.

Conclusion: The presence of a residual tumor after IMRT was a significant negative independent prognostic factor for OS, LRFS and DFS. Although IMRT have improved the distribution of radiotherapy doses into the tumors, residual tumors detected by MRI after IMRT are still associated with poor prognosis in patients with advanced nasopharyngeal carcinoma.
\end{abstract}

Keywords: Nasopharyngeal carcinoma, Residual tumor, MRI, IMRT, Prognosis

\section{Background}

The presence of residual tumors after treatment may provide important prognostic information [1] and may be associated with the radiosensitivity of the tumor. A prospective study of the correlation of regression rate and the probability of recurrence after radiation of neck node metastases in 47 patients by Bartelink showed that tumors with a slow regression rate had a high probability of recurrence [2]. Residual foci detected by clinical or imaging methods may be composed of cancer parenchyma-like

\footnotetext{
* Correspondence: Ifshen2008@163.com

Department of Oncology, Xiangya Hospital, Central South University, Hunan Province, No. 87, Xiangya Road, Changsha, Hunan Province 410008, P.R. China
}

cancer stem cells and delayed reproductive-dead cells, as well as other cell types including interstitial tissue-like fibrosis and mononuclear cells [3]. While reproductivedead cells will disappear within several months, radioresistant cancer stem cells are thought to be the root cause of relapse and metastasis [4], and the radio-response of the interstitial tissue is also associated with recurrence [3]. Given these possibilities, clinicians need to decide whether or not to increase the local dosage or provide timely additional adjuvant chemotherapy for patients with radiographically-visible residual tumors at the end of radiotherapy. Biopsy is the "gold standard" for diagnose of residual tumor. However, most the residual tumors are 
located outside the nasopharynx in the patients with locally-advanced nasopharyngeal carcinoma (NPC) so biopsy for the tumors is difficult to be obtained. $\mathrm{Ng}$ et al. [5] in a retrospectively reviewed study reported the MRI features of recurrent NPC in 72 patients who underwent MRI and showed a nasopharyngeal mass in 50 patients (69.4\%) involved outside the nasopharynx including the parapharyngeal space $(44.4 \%)$, nasal cavity $(12.5 \%)$, paranasal sinuses $(27.8 \%)$, oropharynx $(4.2 \%)$, orbit $(8.3 \%)$, infratemporal fossa (18.1\%), skull base (59.8\%), intracranial area (51.4\%) and regional lymph nodes (15.3\%). Moreover, the viable cells in residual tumors identified by pathology at the end of radiation may become died cells at later time and pathology cannot detect the depth of tumor invasion which is usually a poor indicator for prognosis [6]. Therefore, clinicians must rely on imaging methods to evaluate the therapeutic effect in most cases. Liauw et al. [7] examined the correlation between treatment response and neck dissection pathology, and reported that residual tumors had a negative predictive value of $77 \%$ for complete clinical response and $94 \%$ for radiographic complete response ( $\mathrm{rCR}$ ) in patients with head and neck cancer treated with radiotherapy. Magnetic resonance imaging (MRI) is the preferred modality for determining the extent of soft tissue, perineural infiltration, intracranial spread and skull base invasion of NPC [8]. It was reported that MRI had a higher accuracy for detecting residual and/or recurrent NPC at the primary tumor site than fluorodeoxyglueose positron emission tomography with computed tomography (FDG PET/CT) [9]. Therefore, it is worthy of investigating whether MRI-derived residual tumors are associated with the prognosis of patients with locally-advanced NPC to simplify and improve the diagnosis and treatment of NPC.

The purpose of this retrospective study of 358 patients with locally-advanced NPC was to compare the prognosis of patients with or without residual tumors based on MRI at the end of intensity-modulated radiotherapy (IMRT). The results may provide a basis for assessing the value of boost radiation or timely adjuvant chemotherapy for patients with residual tumors at the end of radiotherapy.

\section{Materials and methods \\ Patients}

A total of 358 patients with locally-advanced NPC (T3/ T4N0-3M0) who received IMRT between August 2008 and December 2011 at Xiangya Hospital of Central South University (Changsha, Hunan province, China) were enrolled in this study. All patients were diagnosed by nasopharyngeal biopsy and nasopharyngeal and neck MRI examinations. In addition to CT/MRI examination of the nasopharynx and neck, the pre-treatment workup also included a complete medical history, physical examination, chest X-ray and/or CT (all patients with N3 disease underwent a chest CT), B-ultrasound scan of the abdomen and neck, bone scan and routine laboratory analysis. The clinical characteristics of the patients are summarized in Table 1. In the study, 354 out of 358 cases were eligible for univariate and multivariate analyses due to 4 patients without MRI review after radiotherapy. Additionally, 346 cases were eligible for survival analysis due to 12 patients loss of follow-up. The study was approved by the ethics committee of

Table 1 Clinical characteristics of the patients and factors associated with residual tumors at the end of IMRT

\begin{tabular}{|c|c|c|c|c|c|}
\hline Characteristic & Group & Patients without residual tumor $(n=212)$ & Patients with residual tumor $(n=142)$ & $x^{2}$ & $P$ \\
\hline \multirow[t]{2}{*}{ Age (y) } & $<50$ & $138(59.7 \% *)$ & $93(40.3 \%)$ & 0.006 & 0.938 \\
\hline & $\geq 50$ & $74(60.2 \%)$ & $49(39.8 \%)$ & & \\
\hline \multirow[t]{2}{*}{ T-stage } & T3 & $42(66.7 \%)$ & $21(33.3 \%)$ & 1.47 & 0.226 \\
\hline & T4 & $170(58.4 \%)$ & $121(41.6 \%)$ & & \\
\hline \multirow[t]{2}{*}{ N-stage } & No-1 & $120(68.2 \%)$ & $56(31.8 \%)$ & 10.02 & 0.002 \\
\hline & N2-3 & $92(51.7 \%)$ & $86(48.3 \%)$ & & \\
\hline \multirow[t]{2}{*}{ Overall stage } & III & $38(66.7 \%)$ & $19(33.3 \%)$ & 1.30 & 0.254 \\
\hline & IV & $174(58.6 \%)$ & $123(41.4 \%)$ & & \\
\hline \multirow[t]{2}{*}{ Chemotherapy } & Yes & $197(59.0 \%)$ & $137(41.0 \%)$ & 2.02 & 0.156 \\
\hline & No & $15(75.0 \%)$ & $5(25.0 \%)$ & & \\
\hline \multirow[t]{2}{*}{ GTVnx V95\% } & $<95 \%$ & $28(37.3 \%)$ & 47 (62.7\%) & 20.15 & $<0.001$ \\
\hline & $\geq 95 \%$ & 184 (65.9\%) & 95 (34.1\%) & & \\
\hline \multirow[t]{2}{*}{ Prescribed dose } & $\leq 73.92 \mathrm{~Gy}$ & 201 (63.0\%) & $118(37.0 \%)$ & 13.09 & $<0.001$ \\
\hline & >73.92 Gy & $11(31.4 \%)$ & $24(68.6 \%)$ & & \\
\hline
\end{tabular}

*Percentage $=$ the number before the bracket divided by the numbers of before the bracket in the same line of column 3 plus column 4. GTVnx: Primary gross target volume; GTVnx V95\%: percentage volume of GTVnx receiving 95\% prescribed doses. 
Xiangya Hospital of Central South University (approval number 201111086).

\section{MRI imaging}

MR imaging was performed with a $1.5-\mathrm{T}$ unit- Siemens Vision Plus (Erlangen, Germany). The protocol was used for the NPC study including axial T1-weighted images without fat saturation, axial T2-weighted images, axial proton density images, sagittal T1-weighted images, and postcontrast axial, coronal and sagittal T1-weighted images with fat saturation. The upper extent covers $2 \mathrm{~cm}$ above the sella turcica and the lower extent reaches $2 \mathrm{~cm}$ below the lower edge of the clavicle. Axial T1weighted fast spin-echo (FSE) images were obtained with repetition time msec/echo time msec of $600 / 15$, echo train length of eight, two signals acquired, 24-mm field of view, $256 \times 256$ matrix, 5-mm-thick section, and 0.5mm gap. Axial T2-weighted FSE images were obtained with 4200/102, echo train length of 16, two signals acquired, 20-mm field of view, 256× 256 matrix, 4-mmthick section, and $0.4-\mathrm{mm}$ gap. An intravenous bolus injection of $0.1 \mathrm{mmol} / \mathrm{kg}$ of body weight gadopentetate imeglumine (Magnevist; Schering, Berlin, Germany) was administered at $2 \mathrm{~mL} / \mathrm{sec}$ for the contrast-enhanced series.

\section{Imaging evaluation}

To reduce subjectivity, all patients were restaged according to the 7th edition of the American Joint Committee on Cancer (AJCC) Staging System for NPC; the pre- and post-treatment MRI scans for each patient were independently reviewed by two senior clinicians from the Departments of Radiology and Oncology. Local radiographic residual tumors were diagnosed by the consensus agreement of two head and neck radiologists and two senior radiation oncologists, respectively. Diagnostic criteria of residual tumors on MRI at different sites as follows: residual tumors present in the nasopharynx or other soft tissues following radiotherapy usually appeared as hypo-intense signal on T1-weighted imaging, as hyper-intensity signal on T2-weighted imaging and also exhibited enhancement following administration of Gd-DTPA. Regional lymph nodes were considered to have residual tumors on MRI if they were larger than $10 \mathrm{~mm}$ in short-axis diameter for cervical lymph nodes and larger than $5 \mathrm{~mm}$ for the retropharyngeal nodes at the end of radiotherapy. For the residual tumors at the skull base on MRI at the end of radiotherapy, we used the reference from previous reports $[10,11]$. It would be considered as residual tumors if the bone of the skull base was destructed with soft tissues and the degree and scope of strengthening of bone have not decrease compared to that of prior to chemoradiotherapy.

\section{Treatment}

All patients underwent IMRT. The target volumes were defined with reference to International Commission on Radiation Units and Measurements (ICRU) reports No. 50 and No. 62. The primary tumor (GTVnx) and positive lymph nodes (GTVnd) were defined and the retropharyngeal lymph nodes were included in the GTVnx. The primary tumor before chemotherapy was delineated as the GTVnx for patients receiving neoadjuvant chemotherapy; two clinical target volumes (CTVs) were defined as follows: CTV1, the high-risk areas including a 5$10 \mathrm{~mm}$ extension around the GTVnx and other high-risk regions such as parapharyngeal space, inferior part of sphenoid sinus, posterior $1 / 3$ of nasal cavity, posterior $1 / 3$ of maxillary sinus, skull base, clivus, oval foramen, lacerated foramen and high-risk lymphatic drainage areas such as retropharyngeal lymph nodes, upper cervical lymph nodes levels II, III, and Va, 60 Gy irradiation was given; and CTV2, the low-risk lymphatic drainage areas including lower cervical lymph nodes levels IV and Vb, 50 Gy irradiation was given. The corresponding planning target volumes (PTVs) were generated by extending each CTV by $3 \mathrm{~mm}$; the prescribed doses for the PGTVnx (GTVnx + $3 \mathrm{~mm}$ margin) were 66.0-75.9 Gy; GTVnd, 69.96-72.6 Gy; PTV1, 59.4-64.0 Gy, PTV2, 50.0-54.0 Gy. The doses to the PTV2 were administered over 28 fractions and other doses over 33 fractions; all patients were treated with simultaneous modulated accelerated radiotherapy once a day for five days a week. Dose limits for the critical tissue structures and plan evaluation were as defined by the Radiation Therapy Oncology Group (RTOG) 0225 [12]. The patients were re-examined by MRI when they finished radiotherapy or the radiation dose reached to approximately $70 \mathrm{~Gy}$. The patient with significant residual tumors at the end of radiotherapy was observed or treated with an IMRT boost dose of 4-10 Gy to the residual lesions over 2-5 fractions depending on the individual toxicity (Organs at risk) and tumor response to radiotherapy. Chemotherapy was part of the treatment plan for all patients; 21 patients who were unwilling to receive chemotherapy or could not tolerate chemotherapy did not undergo chemotherapy. Neoadjuvant chemotherapy was administered when the waiting time for radiotherapy was longer than acceptable or to downsize bulky tumors. At the end of radiotherapy, adjuvant chemotherapy was administered to the patients with N2/N3 stage disease and with existing residual disease detected by MRI or physical examination.

\section{Follow-up}

The follow-up methods included direct telephone calls to the patients or their families; or hospital visits for the patients. Follow-up was measured from the first day of treatment to last follow-up date or date of patient's death. After radiotherapy, follow-up examinations were 
conducted once every 3 months in the first 2 years, once every 6 months in years 2 to 5 , and annually thereafter. MRI of the nasopharynx and neck region was performed once a year for the patient with no residual tumor, or every 3-6 months for the patient with residual tumor.

Recurrence was defined as the tumor regrown after disappearing at least one month. The duration of overall survival (OS) was calculated from the day of radiotherapy completion to the date of patient's death or last follow-up. The duration of local relapse-free survival (LRFS) was calculated from the day of radiotherapy completion to the date of tumor local recurrence. The duration of disease-free survival (DFS) was calculated from the day radiotherapy completion to the date of tumor recurrence, distant metastasis or death.

\section{Statistical analysis}

All statistical analyses were performed using Statistical Package for the Social Sciences version 17.0 (SPSS, Chicago, IL, USA). Actuarial rates were calculated using the Kaplan-Meier method and differences were compared using the log-rank test. Multivariate analysis with the Cox proportional hazards model was used to test for independent significance by backward elimination of insignificant explanatory variables. The Mann-Whitney test was used to examine the between-group differences in the GTVnx V95\% value. The criterion for statistical significance was set at $\alpha=0.05$ and $P$-values were based on two-sided tests.

\section{Results}

\section{Clinical characteristics of the patients}

The clinical characteristics of the patients are presented in Table 1. At the end of radiotherapy, 142/354 cases (40.1\%) had residual tumors. The residual tumor rate for patients with N2-3 disease was significantly higher than that of patients with N0-1 disease $(P=0.002)$. The patients in the residual tumor group had a higher prescribed radiation dose than the patient in no residual tumor group $(P<0.001)$. The residual tumor rate was significantly lower in the patients with the minimum absorbed dose of the 95\% GTVnx (GTVnx D95\%) $\geq 70$ Gy or GTVnx V95\% $\geq 95 \%$ compared to the patients with a GTVnx D95\% $<70$ Gy $(P<0.001)$ or GTVnx V95\% $<95 \%$ $(P<0.001)$, respectively. The locations and numbers of residual tumors are summarized in Table 2. As mentioned previously, only $11.44 \%$ of residual tumors were located in the pharyngonasal cavity, 30.93\% in the skull base, and $30.15 \%$ in the parapharynx and other soft tissues, and $45.07 \%$ of patients had more than one residual tumor.
Table 2 Characteristics of the $\mathbf{2 3 6}$ residual tumors from 142 patients with PNC at the end of IMRT

\begin{tabular}{lll}
\hline Characteristic & Number & Percentage (\%) \\
\hline Residual tumor location & 27 & 11.44 \\
Pharyngonasal cavity & 73 & 30.93 \\
Skull base & 72 & 30.15 \\
Parapharynx and Other soft tissues & 23 & 9.75 \\
Paranasal sinus & 9 & 3.81 \\
Intracranial space & 32 & 13.56 \\
Cervical lymph nodes & 236 & 100.00 \\
Total & & \\
Number of residual tumors* & 78 & 54.93 \\
One tumor & 64 & 45.07 \\
More than one tumor & 142 & 100.00 \\
Total &
\end{tabular}

Number of residual tumors* refers to the number of anatomic sites containing residual tumors including the pharyngonasal cavity, skull base, parapharynx and other soft tissues, intracranial space, and cervical lymph nodes. Some patients had more than one residual tumor.

\section{Treatment outcomes}

The median follow-up period for all patients was 45 months (range, 3-78 months). In total, 22/346 cases developed local recurrence (6.36\%), 55/346 developed distant metastasis (15.9\%), and 9/346 developed recurrence plus distant metastasis (2.6\%). Among the local relapse cases, 4 nasopharyngeal, 5 cervical lymph nodes, 6 bone of the skull base, 1 orbit, 2 paranasal sinus, 2 pharyngeal lymph nodes and 2 parapharyngeal spacerecurrences. Among the distant metastasis cases, 15 liver metastasis, 24 pulmonary metastasis, 20 bone metastasis, 1 adrenal metastasis, 1 retroperitoneal metastasis, and $10 \mathrm{mul}-$ tiple organs metastasis. There were 64 deaths out of 346 patients (18.5\%) which 49 patients were due to tumor recurrence and metastasis, 10 were due to tumor-associated complications ( 5 cases nasopharyngeal hemorrhage, 1 case septic shock, 3 cases malnutrition systemic failure, and 1 case syncope), 1 patient was due to gastrointestinal bleeding and 4 patients were due to unknown causes.

\section{Prognostic value of residual tumors after IMRT}

Survival rates were calculated using the KaplanMeier method and compared using the log-rank test. The 3-year OS rate for the entire cohort study was $83 \%, 73 \%$ in the residual tumor group and $90 \%$ in the no residual tumor group (HR 2.15, 95\% CI 1.213.82, $P=0.007)$; 3-year LRFS for the entire cohort study was $94 \%, 89 \%$ in the residual tumor group and $97 \%$ in the no residual tumor group (HR 4.46, 95\% CI 1.61-12.38, $P=0.002$ ); 3-year DFS for the entire 
cohort study was $76 \%, 67 \%$ in the residual tumor group and $82 \%$ in the no residual tumor group (HR $2.21,95 \%$ CI 1.40-3.48, $P=0.001$ ). Survival curves demonstrated that the presence of a residual tumor was associated with poorer survival outcomes, as shown in Figure 1, the statistically significant differences of the OS, LRFS and DFS survival curves between the residual tumor group and no residual tumor group (OS: $\mathrm{HR}=2.37,95 \%$ CI 1.43-3.93, $P=0.001$; LRFS: $\mathrm{HR}=3.92,95 \%$ CI $1.50-10.28, P=0.003$; DFS: $\mathrm{HR}=2.10,95 \%$ CI 1.36-3.24, $P=0.001)$.

The Univariate analysis suggests that the factors influencing the 3 -year $O S$ rate are age $(P=0.002)$, $\mathrm{N}$-stage $(\mathrm{P}=0.003)$, overall stage $(\mathrm{P}=0.038)$, and presence or absence of a residual tumor at the end of radiotherapy $(P=0.001)$, the factors influencing LRFS are age $(\mathrm{P}=0.001)$ and presence or absence of a residual tumor at the end of radiotherapy $(\mathrm{P}=0.003)$, and the factors influencing DFS are age $(\mathrm{P}=0.008), \mathrm{N}$-stage $(\mathrm{P}=0.024)$, T-stage $(P=0.05)$, overall stage $(P=0.024)$, and presence or absence of a residual tumor at the end of radiotherapy $(\mathrm{P}=$ 0.001). However, chemotherapy and prescribed radiation dose are not the factors for significantly influencing the OS, LRFS, or DFS (Table 3).

The following factors were associated with treatment outcomes in multivariate analysis (Table 4): age (HR 2.32, $P=0.001$ ), N-stage (HR 1.43, $P=0.008$ ), and the presence or absence of a residual tumor after radiotherapy (HR 2.11, $P=0.004$ ) were significantly associated with OS; age (HR 4.80, $P=0.002$ ), and the presence or absence of a residual tumor after radiotherapy (HR 4.80, $P=0.002)$ were significantly associated with LRFS; age (HR 1.81, $P=0.007)$, N-stage (HR 1.26, $P=0.049$ ), and the presence or absence of a residual tumor after radiotherapy (HR 1.91, $P=0.004$ ) were significantly associated with DFS.

\section{Relationship between the radiation dose and the presence of residual tumors at the end of IMRT}

Prescribed radiation doses for all patients in the study were ranged from 62.72 Gy to 80.64 Gy with a midvalue of $73.92 \mathrm{~Gy}$. A higher prescribed dose (>73.92 Gy) was only given to the patients who still had a large residual tumor and the OARs (Organs at risk) were not at
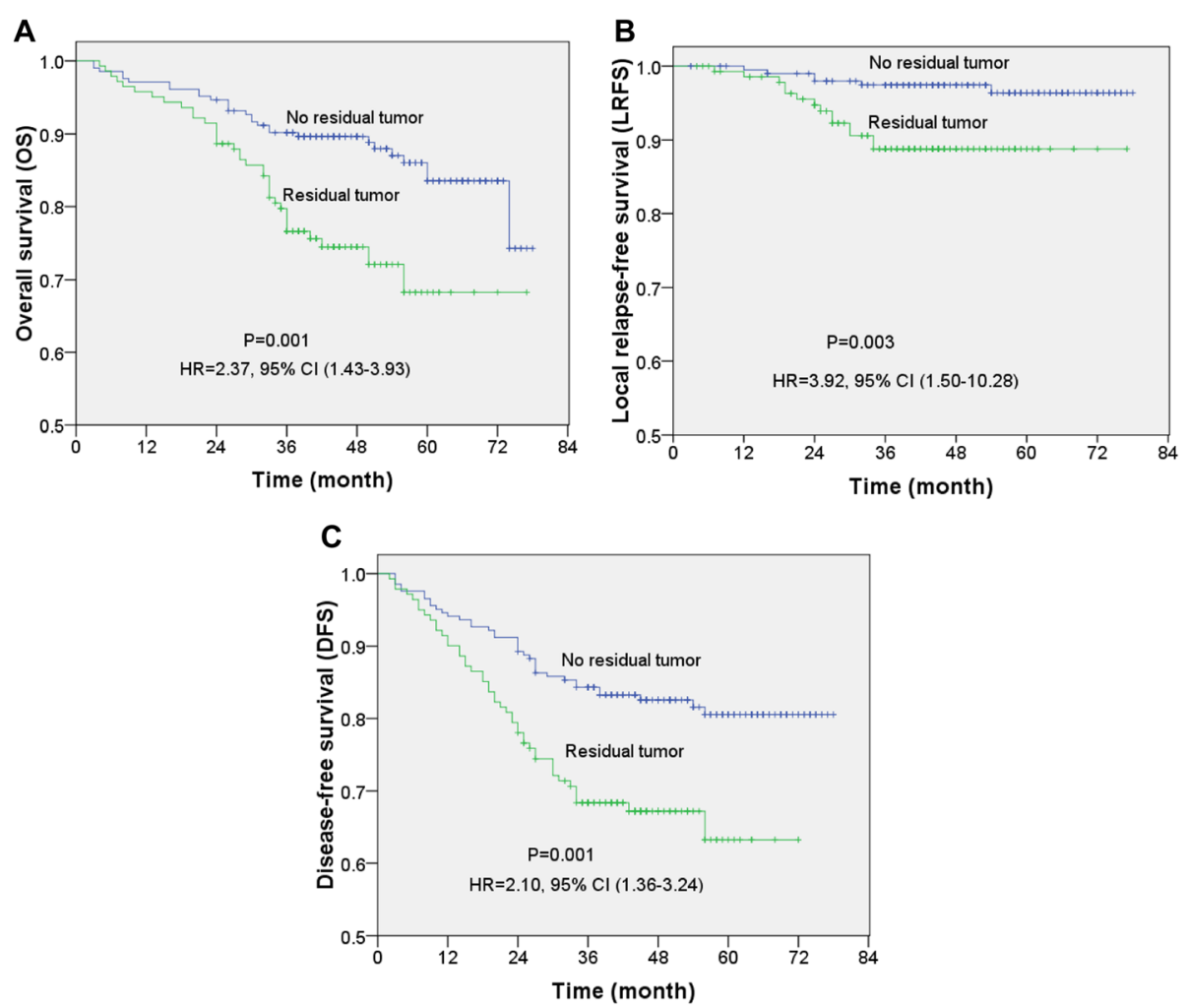

Figure 1 Kaplan-Meir survival curves for 346 patients with locally-advanced nasopharyngeal carcinoma (NPC). (A) Overall survival (OS), (B) local relapse-free survival (LRFS), (C) disease-free survival (DFS) for patients stratified by the presence and absence of a residual tumor at the end of IMRT. P-values were calculated using the unadjusted log-rank test; Hazard ratios (HR) were calculated using the unadjusted Cox proportional hazards model; 95\% Cl: 95\% confidence interval. 
Table 3 Univariate analysis of prognostic factors in patients with NPC

\begin{tabular}{|c|c|c|c|c|c|c|c|}
\hline Variable & No." $\mathrm{N}=346$ & 3-year OS (\%) & p-value & 3-year LRFS (\%) & p-value & 3-year DFS (\%) & $p$-value \\
\hline Age & & & 0.002 & & 0.001 & & 0.008 \\
\hline$<50 y$ & 225 & 87 & & 97 & & 80 & \\
\hline$\geq 50 y$ & 121 & 75 & & 87 & & 69 & \\
\hline $\mathrm{N}$-stage & & & 0.003 & & 0.685 & & 0.024 \\
\hline No & 63 & 92 & & 94 & & 84 & \\
\hline N1 & 110 & 87 & & 92 & & 77 & \\
\hline N2 & 113 & 84 & & 94 & & 77 & \\
\hline N3 & 60 & 67 & & 96 & & 65 & \\
\hline T-stage & & & 0.105 & & 0.838 & & 0.050 \\
\hline T3 & 59 & 90 & & 95 & & 88 & \\
\hline T4 & 287 & 82 & & 93 & & 74 & \\
\hline Overall stage & & & 0.038 & & 0.684 & & 0.024 \\
\hline III & 53 & 92 & & 94 & & 91 & \\
\hline IV & 293 & 82 & & 94 & & 74 & \\
\hline Chemotherapy & & & 0.386 & & 0.059 & & 0.427 \\
\hline Yes & 327 & 84 & & 94 & & 77 & \\
\hline No & 19 & 72 & & 83 & & 68 & \\
\hline Prescribed dose & & & 0.926 & & 0.985 & & 0.793 \\
\hline$\leq 73.92$ Gy & 311 & 83 & & 93 & & 76 & \\
\hline >73.92 Gy & 35 & 85 & & 94 & & 75 & \\
\hline Residual tumor* & & & 0.007 & & 0.002 & & $<0.001$ \\
\hline No & 205 & 90 & & 97 & & 82 & \\
\hline Yes & 141 & 73 & & 89 & & 67 & \\
\hline
\end{tabular}

*Residual tumor: tumor detected by MRI at the end of radiotherapy.

\#No.: number of patients.

OS: overall survival; LRFS: local relapse-free survival; DFS: disease-free survival.

risk to accept radiation boost. However, the data show that increase of radiation doses did not reduce the rate of residual tumor, the residual rates were $68.6 \%$ and $37.0 \%$ for the groups of high prescribed dose and lower prescribed dose, respectively; or did not improve the treatment outcomes (Table 5). The 3-year OS, LRFS, and DFS rates were $83 \%$ vs $85 \%, 93 \%$ vs $94 \%$ and $76 \%$ vs $75 \%$ for the groups of high prescribed dose and low prescribed dose, respectively (Table 3 ). There was not statistically significantly different $(\mathrm{p}>0.05)$. The median

Table 4 Summary of multivariate analysis of prognostic factors in patients with NPC

\begin{tabular}{|c|c|c|c|c|c|c|c|}
\hline \multirow[t]{2}{*}{ End point } & \multirow[t]{2}{*}{ Variable } & \multirow[t]{2}{*}{ Regression coefficient } & \multirow[t]{2}{*}{ Standard error } & \multirow[t]{2}{*}{$\mathrm{HR}$} & \multicolumn{2}{|l|}{$95 \% \mathrm{Cl}$} & \multirow[t]{2}{*}{$P$-value } \\
\hline & & & & & Lower & Upper & \\
\hline \multirow[t]{3}{*}{ OS } & Age & 0.84 & 0.25 & 2.32 & 1.412 & 3.79 & 0.001 \\
\hline & $\mathrm{N}$-stage & 0.36 & 0.14 & 1.43 & 1.10 & 1.87 & 0.008 \\
\hline & Residual tumor & 0.75 & 0.26 & 2.11 & 1.26 & 3.54 & 0.004 \\
\hline \multirow[t]{2}{*}{ LRFS } & Age & 1.57 & 0.50 & 4.80 & 1.78 & 12.83 & 0.002 \\
\hline & Residual tumor & 1.59 & 0.51 & 4.88 & 1.81 & 13.16 & 0.002 \\
\hline \multirow[t]{3}{*}{ DFS } & Age & 0.59 & 0.22 & 1.81 & 1.17 & 2.80 & 0.007 \\
\hline & $\mathrm{N}$-stage & 0.23 & 0.12 & 1.26 & 1.01 & 1.58 & 0.049 \\
\hline & Residual tumor & 0.65 & 0.23 & 1.91 & 1.23 & 2.98 & 0.004 \\
\hline
\end{tabular}

HR: hazard ratio; $\mathrm{Cl}$, confidence interval; $P$-values were calculated using an adjusted Cox proportional hazards model. OS: overall survival; LRFS: local relapse-free survival; DFS: disease-free survival.

The variables were stratified as following: Age: < 50 y vs $\geq 50$ y; N-stage: N0 vs N1vs N2 vs N3; T-stage: T3 vs T4; Residual tumor: having tumor vs no tumor. The table presents only the data with statistical significances. 
Table 5 Relationship of PGTVnx V95\% and prescribed radiation dose and residual tumor after IMRT

\begin{tabular}{lllllll}
\hline Variable & Group & GTVnx V95\% & & & \\
\cline { 3 - 6 } & & Median & Minimum & Maximum & Interquartile range & P-value \\
\hline Prescribed dose & $>73.92$ Gy & 99.1 & 89.9 & 100 & 2.9 & 0.583 \\
& $\leq 73.92$ Gy & 98.7 & 84.4 & 100 & 4.0 & 5.1 \\
Residual tumor & Yes & 97.2 & 84.4 & 100 & 2.5 \\
\hline
\end{tabular}

GTVnx V95\% = the percentage volume of the GTVnx receiving 95\% of the prescribed dose.

GTVnx V95\% values for the higher radiation group and the low dose group were not significantly different (99.1\% vs. $98.7 \%, P=0.583$ ), indicating that the GTVnx V95\% cannot be improved by increasing the radiation dose. However, the median GTVnx V95\% values were significantly different $(97.2 \%$ vs $99.5 \%, P<0.001)$ between the residual tumor group and no residual tumor group, indicating that a low GTVnx V95\% may be associated with the presence of a residual tumor after treatment (Table 5).

\section{Discussion}

The present study reveals that advanced N-stage, a low GTVnx V95\% were associated with a higher risk of a residual tumor in the patients with locally-advanced NPC. The presence of a residual tumor at the end of IMRT was a significant independent factor for OS, LRFS and DFS in the patients with locally-advanced NPC. Additionally, a higher prescribed radiation dose was not associated with a high GTVnx V95\% and did not improve survival outcome.

In this cohort study of 358 patients with NPC, MRI indicated that 142 patients had residual tumors in total of 236 (some patients had more than one tumor) at the end of IMRT. only 27 tumors were located in the nasopharynx while most tumors were located in the skull base, soft tissues or other tissues that cannot be assessed by biopsy (Table 2). Chan et al. [13] reported that deeply seated residual/recurrent tumors beyond the reach of routine nasopharyngeal biopsy are not rare (15.4\%). However, only less than half of the patients had locally advanced diseases in their study. To understand the status of residual tumors at the end of radiation, only imaging tools CT/MRI/PET are suitable to be used. MRI has become the gold standard for NPC diagnosis and therapeutic evaluation due to the high accuracy and economic cost-effectiveness. The specificity of MRI was reported at the range of 44-83\% [14-16]. Post-irradiation inflammatory changes, such as an immature scar, reactive mucosal and submucosal changes or osteoradionecrosis, may interfere with the interpretation of MRI and thus decrease its specificity. In contrast, 18F-FDG PET showed a significantly higher specificity of $93.4 \%$ in the assessment of treatment response and appears less influenced by radiotherapy (RT)-induced inflammation [14]. A systematic review suggests that FDG-PET is the best modality for diagnosis of local residual or recurrent NPC [16]. However, the results may be disputed by other report showing that there was a trend toward greater overall accuracy of MRI over PET/CT in detecting residual and/ or recurrent NPC at the primary site; $92.1 \%$ for MRI and 85.7\% for FDG PET/CT $(\mathrm{P}=0.16)$ [9]. Because the intracranial localizations and the perineural spreads via the foramen ovale, the anterior foramen lacerum, or the pterygopalatine fossa were depicted with MRI only, FDG PET/ CT is not considered as a good method for the study of the intracranial disease due to the physiologically high FDG uptake by the brain.

In the present study,the MRI-derived residual tumor rate was $40.1 \%(142 / 358)$, similar to the study of Han et al. [17] showed the residual tumor rates of $44.2 \%(72 / 196)$ in the patients treated with IMRT and $26.6 \%(52 / 196)$ in the patients treated with conventional radiotherapy (CRT) detected by nasopharyngeal MRI at the end of radiotherapy. Zhang et al. [18] reported that it has been achieved $40.4 \%$ complete response of primary tumor (CRPT), 44.7\% partial response of primary tumor (PRPT), and $14.9 \%$ stable disease of primary tumor (SDPT) at the end of radiotherapy in a study with 188 NPC patients. Lin et al. [19] reported that $50 \%(54 / 108)$ of residual tumors were detected by MRI in the patients with NPC one month after completing radiotherapy. However, Chan et al. reported only $3.6 \%(4 / 112)$ of the patients were found to have residual tumors 3 months after radiotherapy by MRI or PET-CT [13]. The reason for greatly different rates of residual tumors reported above may be due to multiple factors including differences in tumor staging, tumor sites, the use of different diagnostic criteria for MRI, technologies of radiotherapy [IMRT or CRT or intracavitary brachytherapy (ICBT)] and the application of comprehensive treatments and so on. In our study, the results in Table 1 suggest that the presence of residual tumors is associated with the latter N stage and the GTVnx V95\%.

Another important factor is the time point for evaluation of the residual tumors. Tumor response to chemoradiotherapy is time-dependent so a positive histological result after radiotherapy may become negative after 12 weeks [20]. This is why the residual tumor rate is so 
low in the study of Chan et al. and most oncologists prefer to assess the residual tumor at the time of three months after radiotherapy. However, it may reduce the efficacy of chemoradiotherapy at three months after radiotherapy due to delayed treatment for the residual tumor. Early intervention to modify the treatment strategy may improve the treatment outcome for patients with residual tumors. It is still controversial whether or not the tumor regression rate during or after treatment is correlated with the survival rate in the patients with NPC. Wang et al. [21] reported that the patients who achieved a slow response ( $\mathrm{CR}$ when the radiation dose was around 70 Gy) had a significantly better prognosis than the patients who achieved rapid regression (CR when the radiation dose was within 50 Gy). Moreover, the prognosis of both of CR groups was significantly better than the patients with a residual tumor at the end of radiotherapy. However, this study was based on the patients who received conventional radiotherapy. Recently, Zhang et al. [18] found that the 5-year OS rates for the patients with CRPT, PRPT, and SDPT at the end of radiotherapy were $84.0 \%, 70.7 \%$, and $44.3 \%$, respectively $(\mathrm{HR}=2.177, \mathrm{P}<0.001)$. However, approximately one third of the patients had stage T1 or T2 disease and $55.9 \%$ of the patients were treated with conventional 2-dimensional radiotherapy (2D-CRT) in their study. The radiobiological effect of IMRT is different from conventional radiotherapy, residual lesions after IMRT have always been received a radical radiation dose. Dose the residual lesions still have an impact on the survival of the patients with NPC? In our study, all patients undergoing IMRT and residual tumors at the end of radiotherapy are still an important negative prognostic factor for OS (3 year OS was 73\% vs $90 \%$ in the residual tumor group and in the no residual tumor group, $P=0.007$ ), LRFS (3-year LRFS was $89 \%$ vs $97 \%$ in the residual tumor group and in the no residual tumor group, $\mathrm{P}=0.002$ ), and DFS (3-year DFS was $67 \%$ vs $82 \%$ in the residual tumor group and in the no residual tumor group, $P<0.001)$. However, Fang et al. [22] reported that the regression rates for the primary tumors and lymph nodes were not significantly associated with local or regional recurrence and overall survival with radiotherapy at 45 Gy. Mantyla et al. [1] reported that in patients with early-stage head and neck cancer (T1-2N0), a significantly more favorable prognosis was observed if the tumor was disappeared by the mid-point of treatment (30 Gy) than that of tumor disappeared by the end of treatment. On the contrary, the prognosis for patients with advanced disease was significantly more favorable if the tumor was disappeared at the end of radiotherapy (2D-CRT) than that of tumors disappeared by the midpoint of treatment. The longer the time after radiotherapy, the more the tumor will regress. Therefore, it needs more times for tumor regression in locally-advanced tumor than in early-stage tumor. It suggests that it is not a good practice to evaluate curative effect too early for the patients with locally advanced NPC.

The prognosis of patients with NPC is related to numerous clinical factors such as age, clinical stage, chemotherapy, radiation dose, and so on. In our study, age is an independent prognostic factors for $\mathrm{OS}(\mathrm{HR}=2.32, \mathrm{P}=$ $0.001)$, LRFS ( $\mathrm{HR}=4.80, \mathrm{P}=0.002)$, and DFS $(\mathrm{HR}=1.81$, $\mathrm{P}=0.007$ ). It is consistent with the results reported by Erkal et al. [23]. N stage is also an independent prognostic factor for OS $(\mathrm{HR}=1.43, \mathrm{P}=0.008)$ and $\mathrm{DFS}(\mathrm{HR}=1.26$, $P=0.049$ ). Clinic staging had a very certain influence on the prognosis of NPC [24]. However, the influence of T stage on the prognosis of NPC becomes smaller with the application of IMRT. In one of the recent studies, multivariate analyses of 305 patients undergoing IMRT revealed that $\mathrm{T}$-classification had no predictive value for local control and survival, whereas only $\mathrm{N}$-classification was a significant prognostic factor for OS [25]. In our study, T-stage also had no predictive value for OS, and LRFS (Table 3). The 7th edition of the AJCC Staging System was adopted in our study and medial or lateral pterygoid involvement was staged as T4. However, medial and/or lateral pterygoid involvement was staged as T3 or T2 by other studies due to better prognosis compared to skull base involvement which being staged as T3 [26,27]. The reason of $\mathrm{T}$ stage and overall stage has no significant effect on NPC may be caused by the pitfall of the 7th AJCC Staging System. The effect of adjuvant chemotherapy on prognosis has been disputed for a long time, study showed that patients treated with chemotherapy as an adjuvant to radiotherapy had a better DFS compared to the patients without adjuvant chemotherapy $(\mathrm{P}=0.04)$ [28]. Univariate analysis in our study also showed that the rates of 3 year OS ( $84 \%$ vs $72 \%$ ), LRFS ( $94 \%$ vs $83 \%$ ), and DFS (77\% vs 68\%) were slight improved in the chemotherapy group compared to the group without chemotherapy, but the difference was not statistically significant $(P>0.05)$. The results may be affected due to too small numbers with the group without chemotherapy $(\mathrm{n}=19)$.

In the present study, prescribed higher radiation doses did not improved the survival outcome by both univariate and multivariate analyses. It has reported that IMRT has improved the treatment outcome in the patients with NPC [29]. IMRT offers a number of advantages over conventional radiotherapy in the terms of target conformity and the ability to increase the radiation dose to the target volume while sparing the surrounding normal organs at risk [30]. Study from a Hong Kong group found that it was a significant determinant of progression-free survival and distant metastasis-free survival for advanced T-stage tumors when the doses were escalated to above 66 Gy in IMRT-based therapy [31]. However, despite the advancement in IMRT dosimetric inadequacy remains a significant 
problem when a tumor invades directly into critical neurological OARs such as optic chiasm, brainstem and spinal cord [32]. In the present study, most patients who had residual tumors received a radical radiation dose: $67.6 \%$ patients (96/142) received a GTVnx V95\% $\geq 95 \%$ and $83.1 \%$ patients $(118 / 142)$ received a $\mathrm{D} 95 \% \geq 66$ Gy. From the data in Table 5, we can see that the median GTVnx V95\% value for the patients in the residual tumor group (97.2\%) was significantly lower than that of the patients in the no residual tumor group (99.5\%; $P<0.001$ ). This indicates that the GTVnx V95\% is one factor associated with the presence of residual tumors after treatment. However, the median GTVnx V95\% values were not significantly different $(P>0.05)$ between the patients in the group of prescribed high doses $>73.92$ Gy and in the group of low doses $\leq 73.92$ Gy. The data indicate that increase of the prescribed dose cannot improve the GTVnx V95\% and it may due to that the radiation dose in the tumor near the OAR had not been improved, instead of increased radiation dose in the normal tissues to lead to an increased risk of radiotherapy complications. There were 10 deaths from radiotherapy complications in our study. This may be the reason that elevation of prescribed radiation doses cannot improve survival outcome.

In the present study, we demonstrate that the presence of a MRI-derived residual tumor after IMRT is an important negative prognostic factor in the patients with locally-advanced NPC. However, this study has several limitations. First, this investigation was a retrospective analysis. Second, the follow-up time (3-6 years) may be too short to detect a relapse of tumor. Moreover, MRI has some shortcoming, we cannot rule out the possibility that abnormal signals on MRI may be due to a reactive change as a result of radiotherapy without the verification by pathology or PET-CT.

\section{Conclusions}

The presence of a residual tumor detected by MRI at the end of IMRT in patients with locally-advanced NPC is closely associated with a poor prognosis, which suggests a potential role for MRI in predicting local control and prognosis in patients with NPC. Additionally, elevating the prescribed dose after radical radiotherapy did not increase the GTVnx V95\% or improve the outcome of survival in locally-advanced NPC received IMRT treatment.

\section{Competing interests}

The authors declare that they have no competing interests.

\section{Authors' contribution}

LFS and YXH have participated in the research design and conducted the experiments and follow-up. QZ, LS, YJZ, MJL and RW have conducted experiments and follow-up. LFS, YXH, YJZ and SC have performed data analysis. $\mathrm{YXH}$ and SC have written the manuscript. And all authors read and approved the final manuscript.
Authors' information

Dr. Shousong Cao is a Visiting Professor of Xiangya Hospital, Central South University.

\section{Acknowledgments}

This work was partly supported by the National Natural Science Foundation of China (81372792) and Hunan Department of Science and Technology Foundation, China (No. 2012FJ4321)

Received: 7 September 2014 Accepted: 6 April 2015

Published online: 15 April 2015

\section{References}

1. Mantyla M, Kortekangas AE, Valavaara RA, Nordman EM. Tumour regression during radiation treatment as a guide to prognosis. $\mathrm{Br} J$ Radiol. 1979:52:972-7

2. Bartelink H. Prognostic value of the regression rate of neck node metastases during radiatiotherapy. Int J Radiot Oncol Biol Phys. 1983;9:993-6.

3. Yan JH, Xu GZ, Hu YH, Li SY, Lie YZ, Qin DX, et al. Management of local residual primary lesion of nasopharyngeal carcinoma: II. Results of prospective randomized trial on booster dose. Int J Radiat Oncol Biol Phys. 1990:18:295-8.

4. Deng CC, Liang Y, Wu MS, Feng FT, Hu WR, Chen LZ, et al. Nigericin selectively targets cancer stem cells in nasopharyngeal carcinoma. Int J Biochem Cell Biol. 2013:45:1997-2006.

5. Ng SH, Chang JT, Ko SF, Wan YL, Tang LM, Chen WC. MRI in recurrent nasopharyngeal carcinoma. Neuroradiology. 1999;41:862-85.

6. Sham JS, Wei WI, Kwan WH, Chan CW, Kwong WK, Choy D. Nasopharyngeal carcinoma: pattern of tumor regression after radiotherapy. Cancer. 1990;65:216-20.

7. Liauw SL, Mancuso AA, Amdur RJ, Morris CG, Villaret DB, Werning JW, et al. Postradiotherapy neck dissection for lymph node-positive head and neck cancer: the use of computed tomography to manage the neck. J Clin Oncol. 2006;24:1421-7.

8. Zhang SX, Han PH, Zhang GQ, Wang RH, Ge YB, Ren ZG, et al. Comparison of SPECT/CT, MRI and CT in diagnosis of skull base bone invasion in nasopharyngeal carcinoma. Biomed Mater Eng. 2014;24:1117-24.

9. Comoretto $M$, Balestreri L, Borsatti $E_{1}$ Cimitan M, Franchin $G$, Lise M. Detection and restaging of residual and/or recurrent nasopharyngeal carcinoma after chemotherapy and radiation therapy: comparison of MR imaging and FDG PET/CT. Radiology. 2008;249:203-11.

10. Ng SH, Chan SC, Yen TC, Liao CT, Chang JT, Ko SF, et al. Comprehensive imaging of residual/recurrent nasopharyngeal carcinoma using whole-body MRI at 3 T compared with FDG-PET-CT. Eur Radiol. 2010;20:2229-40.

11. Liang FY, Sun W, Han P, Lu X, Lian YN, Huang XM. Detecting plasma Epstein Barr virus DNA to diagnosis postradiation nasopharyngeal skull base lesions in nasopharyngeal carcinoma patients: a prospective study. Chin J Cancer. 2012;31:142-9.

12. Radiation Therapy Oncology Group RTOG protocol 0225. A phase II study of intensity modulated radiation therapy (IMRT) +/- chemotherapy for nasopharyngeal cancer. Available at: www.rtog.org. Last accessed on July 15, 2014.

13. Chan SC, Ng SH, Chang JT, Lin CY, Chen YC, Chang YC, et al. Advantages and pitfalls of 18F-fluoro-2-deoxy-D-glucose positron emission tomography in detecting locally residual or recurrent nasopharyngeal carcinoma: comparison with magnetic resonance imaging. Eur J Nucl Med Mol Imaging. 2006;33:1032-40.

14. Yen RF, Hung RL, Pan MH, Wang YH, Huang KM, Lui LT, et al. 18-fluoro-2deoxyglucose positron emission tomography in detecting residual/recurrent nasopharyngeal carcinomas and comparison with magnetic resonance imaging. Cancer. 2003;98:283-7.

15. Ng SH, Liu HM, Ko SF, Hao SP, Chong VF. Posttreatment imaging of the nasopharynx. Eur J Radiol. 2002;44:82-95.

16. Liu T, Xu W, Yan WL, Ye M, Bai YR, Huang G. FDG-PET, CT, MRI for diagnosis of local residual or recurrent nasopharyngeal carcinoma, which one is the best? A systematic review. Radiother Oncol. 2007;85:327-35.

17. Han F, Xiao WW, Wang HY, Huang Y, Deng ML, Zhao C, et al. Influence of intensity-modulated radiotherapy on tumor regression in nasopharyngeal carcinoma. Chin J Radiol Med Pr. 2012;32:204-6. 
18. Zhang N, Liang SB, Deng YM, Lu RL, Chen HY, Zhao H, et al. Primary tumor regression speed after radiotherapy and its prognostic significance in nasopharyngeal carcinoma: a retrospective study. BMC Cancer. 2014;14:136

19. Lin GW, Wang LX, Ji M, Qian HZ. The use of MR imaging to detect residual versus recurrent nasopharyngeal carcinoma following treatment with radiation therapy. Eur J Radiol. 2013;82:2240-6.

20. Kwong DL, Nicholls J, Wei WI, Chua DT, Sham JS, Yuen PW, et al. The time course of histologic remission after treatment of patients with nasopharyngeal carcinoma. Cancer. 1999;85:1446-53.

21. Wang XS, Hu CS, Wu YR, Feng Y. Influence of speed in tumor regression on prognosis of nasopharyngeal carcinoma. Chinese J of Radiat Oncol. 2005;14:6-9.

22. Fang FM, Tsai WL, Go SF, Ho MW, Wu JM, Wang CJ, et al. Implications of quantitative tumor and nodal regression rates for nasopharyngeal carcinomas after 45 Gy of radiotherapy. Int J Radiat Oncol Biol Phys. 2001;50:961-9.

23. Erkal HS, Serin M, Cakmak A. Nasopharyngeal carcinomas: analysis of patient, tumor and treatment characteristics determining outcome. Radiother Oncol. 2001;61:247-56.

24. Chen L, Mao YP, Xie FY, Liu LZ, Sun Y, Tian L, et al. The seventh edition of the UICC/AJCC staging system for nasopharyngeal carcinoma is prognostically useful for patients treated with intensity-modulated radiotherapy from an endemic area in China. Radiother Oncol. 2012;104:331-7.

25. Han L, Lin SJ, Pan JJ, Chen CB, Zhang Y, Zhang XC, et al. Prognostic factors of 305 nasopharyngeal carcinoma patients treated with intensity-modulated radiotherapy. Chin J Cancer. 2010;29:145-50.

26. Luo DH, Yang J, Qiu HZ, Shen T, Chen QY, Huang OY, et al. A new T classification based on masticator space involvement in nasopharyngeal carcinoma: a study of 742 cases with magnetic resonance imaging. BMC Cancer. 2014;14:653.

27. Sze H, Chan LL, Ng WT, Hung AW, Lee MC, Chang AT, et al. Should all nasopharyngeal carcinoma with masticator space involvement be staged as T4? Oral Oncol. 2014;50:1188-95.

28. Ozyar E, Gurkaynak M, Yildiz F, Atahan IL. Non-metastatic stage IV nasopharyngeal carcinoma patients: analysis of the pattern of relapse and survival. Radiother Oncol. 2004;72:71-7.

29. Lai SZ, Li WF, Chen L, Luo W, Chen YY, Liu LZ, et al. How does intensity-modulated radiotherapy versus conventional two-dimensional radiotherapy influence the treatment results in nasopharyngeal carcinoma patients? Int J Radiat Oncol Biol Phys. 2011;80:661-8.

30. Miles EA, Clark CH, Urbano MT, Bidmead M, Dearnaley DP, Harrington KJ, et al. The impact of introducing intensity modulated radiotherapy into routine clinical practice. Radiother Oncol. 2005;77:241-6.

31. Kam MK, Teo PM, Chau RM, Cheung KY, Choi PH, Kwan WH, et al. Treatment of nasopharyngeal carcinoma with intensity-modulated radiotherapy: the Hong Kong experience. Int J Radiat Oncol Biol Phys. 2004:60:1440-50

32. Nishioka T, Shirato H, Kagei K, Abe S, Hashimoto S, Ohmori K, et al. Skull-base invasion of nasopharyngeal carcinoma: magnetic resonance imaging findings and therapeutic implications. Int J Radiat Oncol Biol Phys. 2000;47:395-400

\section{Submit your next manuscript to BioMed Central and take full advantage of:}

- Convenient online submission

- Thorough peer review

- No space constraints or color figure charges

- Immediate publication on acceptance

- Inclusion in PubMed, CAS, Scopus and Google Scholar

- Research which is freely available for redistribution 